\title{
Multiple Angiomyolipomata of the Liver: A Case Report
}

Laura H. Tang, M.D., Ph.D., Pei Hui, M.D., Ph.D., Guadalupe Garcia-Tsao, M.D., Ronald R. Salem, M.D., Dhanpat Jain, M.D.

Department of Anatomic Pathology (LHT, PH, DJ), Department of Internal Medicine (GG-T), Division of Digestive Diseases, and Department of Surgery (RRS), Yale University School of Medicine, New Haven, Connecticut

Angiomyolipoma (AML) is a rare benign tumor that occurs most commonly in the kidney. Occasionally it may be found in the liver. Lesions in the liver are usually solitary. Multiple AMLs are extremely rare and are typically seen in patients with tuberous sclerosis. We now report an unusual case of a 46year-old woman with multiple hepatic AMLs. There were more than 15 lesions distributed predominantly in the right hepatic lobe. The tumors ranged from 0.2 to $6 \mathrm{~cm}$ in size and consisted of a variable admixture of proliferating blood vessels, adipose tissue, and smooth muscle. There was no clinical evidence of tuberous sclerosis in this patient. Polymerase chain reaction amplification of the highly polymorphic human androgen receptor gene (HUMARA) was performed and the pattern of $X$ chromosome inactivation was analyzed. Three of the five representative AML nodules showed a preferential loss of one of the two HUMARA alleles indicating a clonal proliferation with involvement of different alleles. Histologic examination of the corresponding lesions showed clonal lesions to be predominantly composed of epithelioid myoid cells while the polyclonal lesions were predominantly composed of adipose tissue. While the histologic diagnosis of AML in a surgical resection specimen is often straightforward, the radiographic, cytologic and intraoperative interpretation of a case with multiple lesions presents a considerable challenge.

KEY WORDS: Angiomyolipoma, Liver, Multiple. Mod Pathol 2002;15(2):167-171

Copyright $(\odot 2002$ by The United States and Canadian Academy of Pathology, Inc.

VOL. 15, NO. 2, P. 167, 2002 Printed in the U.S.A.

Date of acceptance: October 29, 2001.

Address reprint requests to: Dhanpat Jain, M.D., Department of Anatomic Pathology, Yale University of School of Medicine, 310 Cedar Street, New Haven, CT 06511; e-mail: Dhanpat.Jain@yale.edu.
Ishak first described angiomyolipoma (AML) in 1976 as a rare benign mixed mesenchymal tumor of the liver (1). Like its more commonly encountered renal counterpart, AML of the liver consists of a variable admixture of three histologic components, namely blood vessels, smooth muscle and adipose tissue. More than 80 cases of hepatic AMLs have been reported and their clinicopathologic features have been well described (2-9). Most hepatic AMLs are solitary tumors. Multiple AMLs are uncommon and usually occur in tuberous sclerosis patients (2, $10,11)$. There have been only two reported cases of multiple hepatic AMLs in patients without tuberous sclerosis $(12,13)$; both patients were Japanese and each had only two hepatic lesions. To the best of our knowledge, cases with more than two AMLs of the liver in patients without tuberous sclerosis have not been reported to date. We now describe a case of a 46-year-old woman without any evidence of tuberous sclerosis and with more than 15 hepatic AMLs involving the right hepatic lobe at presentation.

\section{CASE REPORT}

\section{Clinical History and Presentation}

The patient is a 46-year-old white woman who had been found to have liver masses 5 years previously in 1995 during a preoperative evaluation of an ovarian mass. She subsequently underwent total abdominal hysterectomy with bilateral salpingooophorectomy for this large ovarian mass, which was found to be an endometrioma she had had thyroidectomy 20 years earlier for a papillary carcinoma of the thyroid. CT scan and MRI revealed at least six masses located in the right lobe of the liver (Fig. 1). The largest lesion measured $4 \mathrm{~cm}$ in greatest dimension while the remaining lesions ranged from less than $1 \mathrm{~cm}$ to $2.2 \mathrm{~cm}$. The smaller four lesions were located superficially at the dome of the right lobe and the rest were deeper within the hepatic parenchyma. The lesions were hypointense on $\mathrm{T} 1$ weighted images and hyperintense on $\mathrm{T} 2$ 


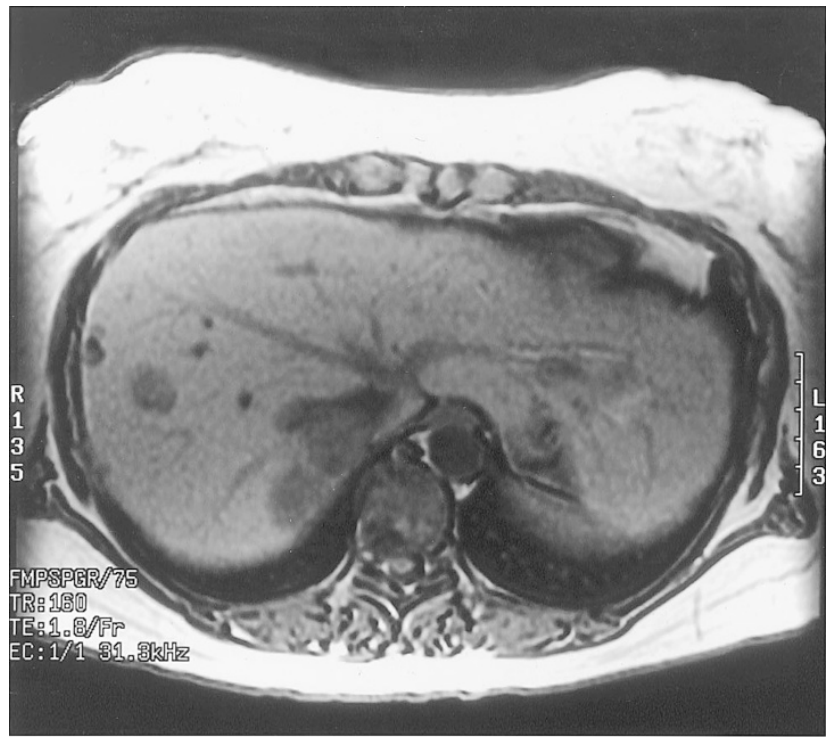

FIGURE 1. The MRI showing hypointense lesions predominantly in the right hepatic lobe.

weighted images. The larger lesions were mildly heterogeneous in signal intensity. The remaining hepatic parenchyma was unremarkable. The differential diagnoses considered were multiple hepatic adenomata and hepatocellular carcinoma. Focal nodular hyperplasia was ruled out with an hepatic HIDA scan.

The patient was entirely asymptomatic with normal liver enzyme levels and no clinical or laboratory findings to suggest underlying chronic liver disease. She admitted using oral contraceptives from age 18 to 22. A fine needle aspiration (FNA) of the lesions was performed yielding abundant cytological material that was interpreted as benign hepatocytes. Given the relative stability of the lesions a benign diagnosis was favored, and the patient was managed conservatively by periodic MRI to assess the growth of these lesions. Over the next $31 / 2$ years, the largest lesion increased to $5.7 \mathrm{~cm}$ in the greatest dimension. Of particular concern was the location of this lesion immediately posterior to and abutting the inferior vena cava. The patient underwent right hepatic lobectomy. Frozen section performed during surgery was interpreted as suggestive of a hepatocytic neoplasm however, the final diagnosis was deferred to permanent sections. Intraoperative ultrasound examination showed no lesions in the left lobe however, MRI performed 5 months after the surgery showed three enhancing lesions (each less than $1 \mathrm{~cm}$ ) in the medial and lateral aspects of the left hepatic lobe.

\section{Pathologic Findings}

\section{Gross}

The right hepatic lobectomy specimen weighed 780 grams and measured $20 \times 12 \times 7 \mathrm{~cm}$. Serial sectioning of the specimen revealed multiple poorly circumscribed, unencapsulated, nodular, yellow to tan lesions ranging from 0.2 to $6 \mathrm{~cm}$ in greatest dimension (Fig. 2). At least 15 lesions were identified grossly. Five were greater than $3 \mathrm{~cm}$ in diameter and showed varying degrees of hemorrhage. The largest lesion measured $6 \times 4 \times 4 \mathrm{~cm}$, was subcapsular in location and extended to the resection margin grossly. This lesion showed extensive hemorrhage and focal necrosis. The smaller lesions had a more yellow-tan and fatty appearance. The surrounding liver parenchyma was unremarkable, and there was no evidence of cirrhosis.

\section{Histopathology}

The lesions were unencapsulated and had an infiltrating border into the adjacent hepatic parenchyma. They were composed of a variable proportion of adipose tissue, thick walled vessels and smooth muscle. Some of the smaller lesions demonstrated a typical histology with good admixture of all three components(Fig. 3A). In the typical lesions the smooth muscle component had a spindle cell morphology however, in many lesions, the smooth muscle cells showed epithelioid features simulating hepatocytes. In fact, some of the larger lesions were predominantly composed of such epithelioid myoid cells arranged in diffuse sheets (Fig. 3B). The epithelioid myoid cells were polygonal in shape, focally showed trabecular arrangement and contained pale eosinophilic granular cytoplasm. In many cells the cytoplasm showed peripheral clearing with radiating strands of cytoplasm sticking to the nucleus producing a "spider cell" appearance. Hyaline cytoplasmic globules and intranuclear cytoplasmic pseudo-inclusions were present in many cells. Moderate nuclear pleomorphism, prominent nucleoli and rare mitotic figures were also identified. Review of the FNA slides revealed that most of

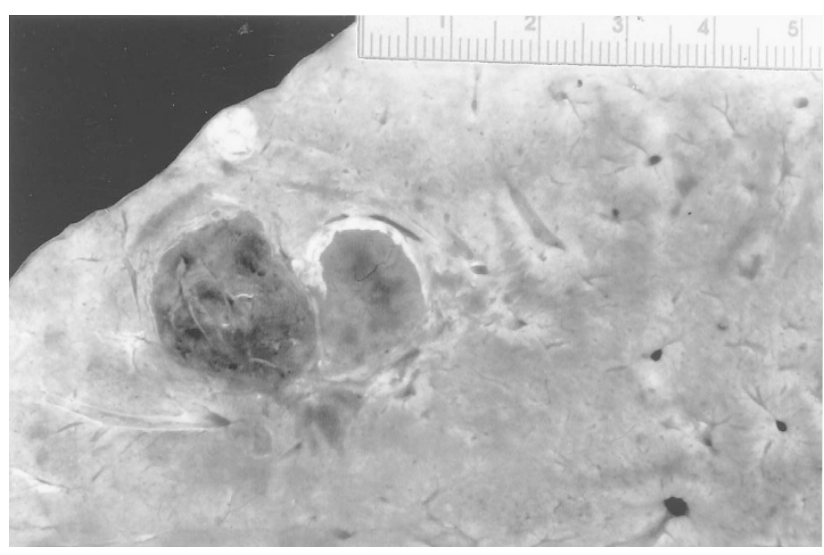

FIGURE 2. Gross photograph of the liver showing three different lesions. The smaller one shows a predominant component of adipose tissue while the two larger ones that were composed of a predominant myoid component show varying degrees of hemorrhage. 

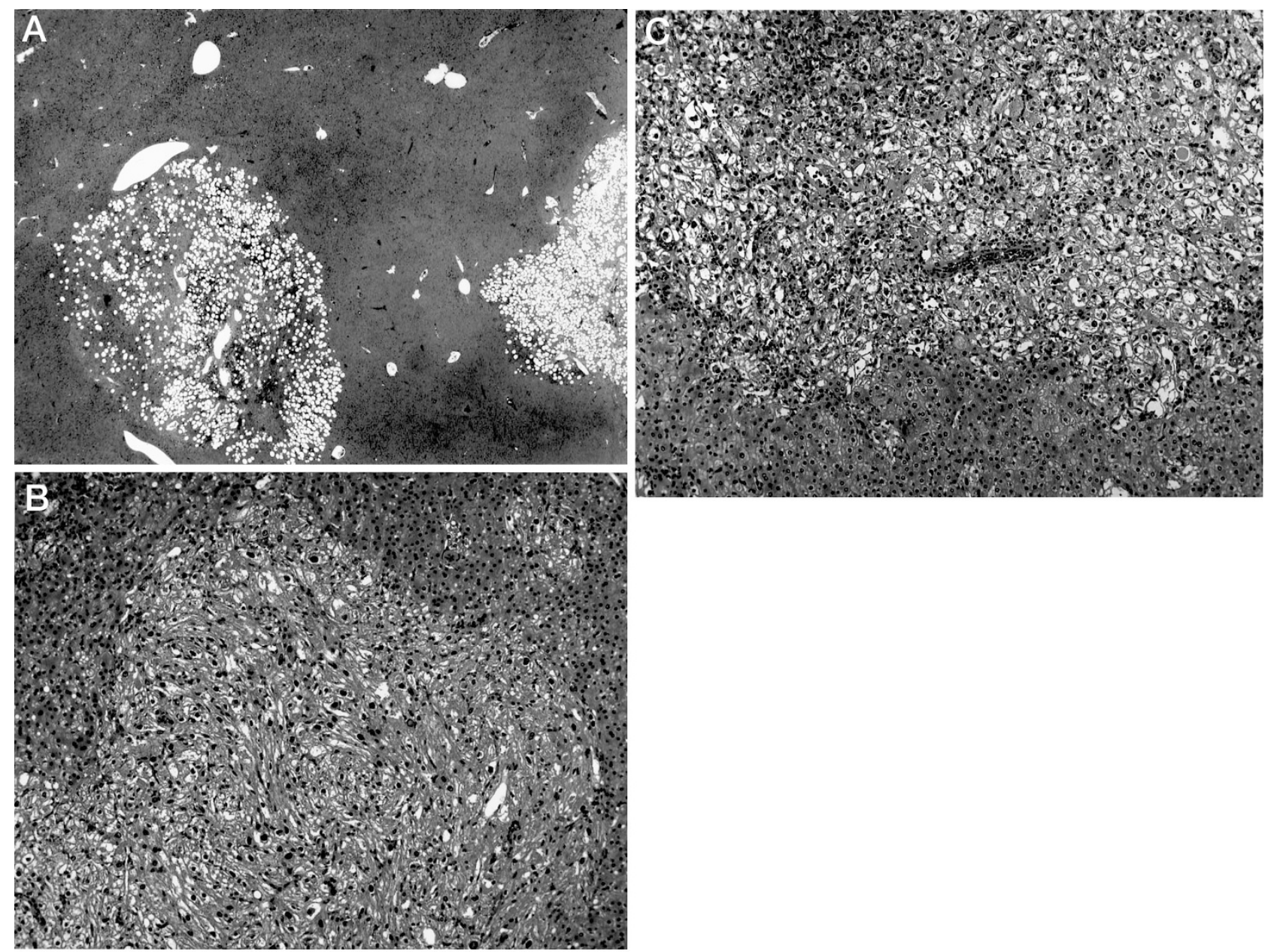

FIGURE 3. A, Low magnification showing two small adjacent angiomyolipomata demonstrating typical histology. B, Higher magnification from one of the larger lesions showing predominant myoid component with spindle cell morphology. C, Higher magnification from one of the larger lesions showing predominant myoid component with epithelioid cell morphology.

the cells initially interpreted as "benign hepatocytes" were in retrospect the epithelioid myoid cell component of the AML. Foci of extramedullary hematopoiesis, varying degree of hemorrhage, numerous hemosiderin-laden macrophages and occasional fibrin thrombi were seen in many of the lesions. The surrounding hepatic parenchyma showed patchy mild steatosis and focal nonspecific portal triaditis. Immunostains performed on the resection specimen demonstrated the epithelioid myoid cells to be strongly positive for HMB45 (1: 400; DAKO, Carpinteria, CA), and negative for keratin AE1/3 (1:2400, Boehringer Mannheim, Indianapolis, IN), CAM5.2 (1:20; Becton Dickinson, San Jose, CA) and desmin (1:2000; DAKO, Carpinteria, CA). Focal and weak area of positive staining for vimentin (1: 10; Biognex, San Vermont, CA) and smooth muscle actin $(1 ; 10000$; Sigma, St. Louis, MO) was found in the epithelioid myoid cells. The vascular smooth muscle and focally the spindle smooth muscle component of the AMLs was strongly immunoreactive for smooth muscle actin, vimentin and desmin.
Electron microscopy demonstrated presence of many mitochondria, abundant rough endoplasmic reticulum, glycogen and rare premelanosome like structures in the cytoplasm of the epithelioid myoid cells. Tight junctions and basal lamina were identified. Thick filaments were not seen in the epithelioid myoid cells.

\section{Clonality Analysis}

As the patient is female, we were provided with an unique opportunity to perform clonality assays on the hepatic AMLs by examining the pattern of $X$ chromosome inactivation. Polymerase chain reaction (PCR) amplification of the highly polymorphic human androgen receptor gene (HUMARA) was performed using methylation-sensitive restriction enzyme, HhaI (14-16). The DNA was extracted from $10 \mu \mathrm{m}$ thick sections representative of various lesions as well as adjacent non-neoplastic liver obtained from formalin fixed paraffin embedded tissue. The amplification product was electrophoresed through a $11 \%$ agarose gel (Fig. 4). Three of 
the five AML nodules showed a preferential loss of one of the two HUMARA alleles indicating a clonal proliferation. Different alleles were involved in these three lesions. The fast moving allele was involved in two of the lesions (Fig. 4, no. 1 and 5), while the slower moving allele was involved in another lesion (Fig. 4, no. 3), as seen on the gel. These results suggest that these tumors originated independently and did not represent intrahepatic spread from a single original clone. Histologic examination revealed that the clonal lesions were predominantly composed of epithelioid myoid cells while the polyclonal lesions were predominantly composed of adipose tissue.

\section{DISCUSSION}

Angiomyolipoma of the liver is an extremely rare benign tumor (1-9). Although the radiologic, histologic, immunohistochemical and ultrastructural features are well described in the literature, the lesions are frequently misdiagnosed. This is often due to a lack of familiarity with the wide clinicopathologic spectrum of these rare lesions.

The association of these tumors with tuberous sclerosis is well established and bilateral or multiple renal AMLs are often associated with tuberous sclerosis (40\%) (17). The incidence of tuberous sclerosis with hepatic AML is only about 6 to $10 \%(2,18)$ and the majority of the hepatic lesions are solitary. Truly multiple AMLs (more than two lesions) along with multiple renal AMLs have only been described in patients with tuberous sclerosis $(2,10,11)$. Two patients, each with two hepatic AMLs, have been reported from Japan without any clinical evidence of tuberous sclerosis $(12,13)$. This is the first report of a patient with multiple hepatic AMLs without neurologic manifestations or any radiologic features suggestive of tuberous sclerosis.

On MRI studies, the fatty component of AML usually demonstrates increased intensity on T1weighted images. The MRI evaluation of our case demonstrated the heterogeneity in one of the larger lesions with low intensity signal on T1-weighted images and high signal on T2-weighted images.

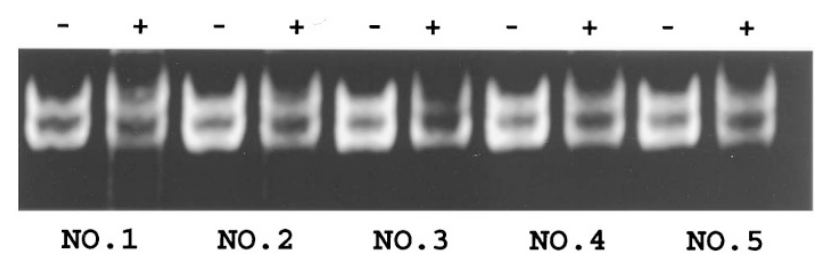

FIGURE 4. Pattern of X-chromosome inactivation as seen by PCR amplification of the human androgen receptor gene showing loss of one of the alleles in three of the lesions (lanes 1, 3, and 5). A nonclonal pattern is seen in two of the lesions (lanes 2 and 4) In each lane the PCR before restriction enzyme digestion is designated by (-) and after the digestion is designated by $(+)$.
These findings suggested the presence of central necrosis. Due to increasing size and its unusual presentation the possibility of AML was not considered. Even the FNA and the intra-operative frozen sections from this lesion which were composed predominantly of the epithelioid myoid cell component were misinterpreted as "hepatocytic lesion."

The clinical differential diagnosis of hepatic AML often includes lipoma, hemangioma, focal nodular hyperplasia, hepatic adenoma and hepatocellular carcinoma. AMLs are usually slow growing and can be followed conservatively. Rapid change in size and composition has only rarely been documented in hepatic AML, and could result from intratumoral hemorrhage, necrosis or actual proliferation of the tumor cells (19). In any given case this could easily raise concern leading to a surgical resection. In our case there was evidence of enlargement of the lesions during the three and a half-year period before the resection. Histologic examination revealed an absence of significant mitotic activity, suggestive of a slow rate of proliferation and raising the possibility that the increase in size was secondary to intratumoral hemorrhage The presentation of hemorrhage or necrosis in an AML is itself an extremely uncommon phenomenon and presents another potential source of confusion with more aggressive neoplasms (2).

Angiomyolipoma exhibit a wide histologic spectrum and increasing awareness of the diverse clinicopathological presentation of these lesions is necessary if surgical resection is to be avoided (20-22). Features that could lead to misinterpretation of AMLs have been discussed in an excellent paper by Tsui et al. (2). In our case, features that were misleading both on the frozen section and the FNA were: 1) sampled areas were composed predominantly of epithelioid myoid cells, 2) the polygonal shape and cytoplasmic clearing of the epithelioid myoid cells simulated hepatocytes, 3) cytoplasmic hyaline globules similar to those often seen in hepatocytic neoplasms were present in epithelioid myoid cells, and 4) presence of adipose tissue in the tumor simulated macrovesicular steatosis on frozen section. Features helpful in making the correct diagnosis were 1) marked variation in the shape and size of the epithelioid myoid cells and their nuclei as compared to normal hepatocytes, 2) foci of spindle cells admixed with epithelioid myoid cells, 3) presence of thick walled tortuous vessels, not typically seen in hepatocytic neoplasms, 4) the lack of an easily identifiable trabecular architecture like that commonly seen in hepatic adenoma and well differentiated hepatocellular carcinoma, 5) the immunoreactivity of the myoid component against HMB45 and smooth muscle actin, and lack of immunoreactivity for keratins (AE1/AE3 and Cam5.2), and finally 6) presence of premelanosome-like 
structure in the epithelioid myoid cells on electron microscopy.

AMLs have been regarded as hamartomas; however, clonality assays performed in a recent study of renal AMLs has shown the myoid and the vascular components to be monoclonal, implying a neoplastic proliferation $(15,16)$. The adipose tissue component of the AML was found to be polyclonal and the authors speculate that the fat is metaplastic or a reactive change in the neoplasm. The findings in our case are similar in that lesions with predominant muscle components (epithelioid or spindle cell) were found to be clonal; further supporting the neoplastic origin of AMLs. Moreover, multiple AMLs in our case seem to represent separate individual clones rather than intrahepatic metastasis.

Another interesting aspect of this case is that the lesions were restricted to the right hepatic lobe at presentation. Postoperative follow-up 5 months after the resection revealed appearance of tiny new lesions in the remaining left lobe. Preferential involvement of only one of the hepatic lobes has been known to occur, not only in cases of hepatic abscesses but also in diffuse process like viral hepatitis. Differential blood flow has been implicated for this phenomenon. Whether this explanation also applies in this case remains a matter of speculation.

In summary, we have reported an unusual case of multiple AML of the liver that presented diagnostic difficulties during preoperative imaging studies, FNA and intra-operative frozen sections. Careful examination of the histologic, immunohistologic and ultrastructural features was helpful in making a correct diagnosis. This case further broadens the wide spectrum of various clinicopathological aspects of AML $s$ and provides some insight into the pathogenesis of multiple AMLs.

\section{REFERENCES}

1. Ishak KG. Mesenchymal tumors of the liver. In: Okuda K, Peters RL, editors. Hepatocellular carcinoma. New York: John Wiley; 1976. p. 247-304

2. Tsui WMS, Colombari R, Portmann BC, et al. Hepatic angiomyolipoma: a clinicopathologic study of 30 cases and delineation of unusual morphologic variants. Am J Surg Pathol 1999;23:34-48.

3. Nonomura A, Mizukami Y, Kadoya M. Angiomyolipoma of the liver: a collective review. J Gastroenterol 1994;29:95-105.

4. Kawarada Y, Mizumoto R. Angiomyolipoma of the liver. Am J Gastroenterol 1983;78:434-9.
5. Takayasu KY, Shima Y, Muramatsu N, Moriyama T. Characteristics of large lipoma and angiomyolipoma of the liver. Cancer 1987;59:916-21.

6. Nguyen GK, Catzavelos C. Solitary angiomyolipoma of the liver. Acta Cytologica 1990;34:201-4.

7. Okada K, Yokoyama S, Nakayama I, Tada I, Kobayashi M. An electron microscopic study of hepatic angiomyolipoma. Acta Pathol Jpn 1989;39:743-9.

8. Fobbe F, Hamm B, Schwarting R. Angiomyolipoma of the liver: CT, MR, and ultrasound imaging. J Comput Assist Tomogr 1988;12:658-9.

9. Linton PL, Ahn WS, Schwartz ME, Miller CM, Thung SN. Angiomyolipoma of the liver: immunohistochemical study of a case. Liver 1991;11:158-61.

10. Hirasaki S, Koide N, Ogawa H, Ujike K, Shinji T, Tsuji T. Tubrerous sclerosis associated with multiple hepatic lipomatous tumors and hemorrhagic renal angiomyolipoma. Int Med 1999;38:345-8.

11. Koide O, Matsuzaka K, Tanaka Y. Multiple giant angiomyolipomas with a polygonal epithelioid cell component in tuberous sclerosis: an autopsy case report. Pathol Int 1998;48: 998-1002.

12. Nonomura A, Mizukami Y, Kadoya M, Takayanagi N, Hirono T. Multiple angiomyolipoma of the liver. J Clin Gastroenterol 1995;20:248-51.

13. Kyokane T, Akita Y, Katayama M, Sato T, Shichino S, Nimura Y. Multiple angiomyolipomas of the liver (case report). Hepato-gastroenterology 1995;42:510-5.

14. Mutter G, Chaponot ML, Fletcher JA. A polymerase chain reaction assay for non-random $\mathrm{X}$ chromosome inactivation identifies monoclonal endometrial cancers and precancers. Am J Pathol 1995;146:501-8.

15. Green AJ, Sepp T, Yates JR. Clonality of tuberous sclerosis hamartomas shown by non-random X-chromosome inactivation. Hum Genet 1996;97:240-3.

16. Saxena A, Alport EC, Custead S, Skinnider LF. Molecular analysis of clonality of sporadic angiomyolipoma. J Pathol 1999;189:79-84.

17. Bissada N, White H, Sun C, Smith PL, Barbour GL, Redman JF. Tuberous sclerosis complex and renal 5 angiomyolipoma. Urology 1975;6:105-13.

18. Robinson JD, Grant EG, Haller JO, Cohen HL. Hepatic angiomyolipomas in tuberous sclerosis. J Ultrasound Med 1989;8:575-8.

19. Irie H, Honda H, Kuroiwa T, Yoshimitsu K, Tajima T, Jimi M, et al. Hepatic angiomyolipoma: report of changing size and internal composition on follow-up examination in two cases. J Comput Assist Tomogr 1999;23:310-3.

20. Nonomura A, Mizukami Y, Muraoka K, Yajima M, Oda K. Angiomyolipoma of the liver with pleomorphic bizarre histologic features. Histopathol 1994;24:279-81.

21. Miyahara M, Kobayashi M, Tada I, Okada K, Sato K, Kim YI, et al. Giant hepatic angiomyolipoma simulating focal nodular hyperplasia. Jpn J Surg 1988;18:346-50.

22. Tsui WM, Yuen AK, Ma KF, Tse CC. Hepatic angiomyolipoma with a deceptive trabecular pattern and HMB-45 reactivity. Histopathology 1992;21:569-73. 